\title{
Dimensions of Subjective Age Identity Across the Lifespant
}

Adults are Aging Physically in Earth Years \& Mentally in Martian Years

Nicole M. Lindner \& Brian A. Nosek

University of Virginia

\section{Background}

As implied by aphorisms like "you're only as old as you eel," subjective age experiences may elicit aspects of age dentity distinct from chronological age. Subjective age has been used in gerontological research to assess age dentity, and it predicts psychological and physical funclioning better than chronological age (Kaufman \& Elder, 2002; Mon

Whereas a slowed subjective experience of time is associated with depression and poor psychological of aging, from a life span perspective, is associated with positive psychological and physical functioning.

Social factors, most notably establishing an identity and (Arnett, 2000), and the link between youth and vitality for alder people, might motivate differential desires to be older among the young, and younger among the old. At the same time, subjective experience of aging may have a psychological reality that is just slower than the

chronological passage of time. We examined how felt age and desired age differed from chronological age across the age span.

\section{Method}

Participants

A convenience sample of 33,751 visitors to the Project Implicit demonstration Web site (http://implicit.harvard.edu) reported:

Chronological Age

2800 Range: $10-89$ years

을 $_{2100}^{2100}$

离

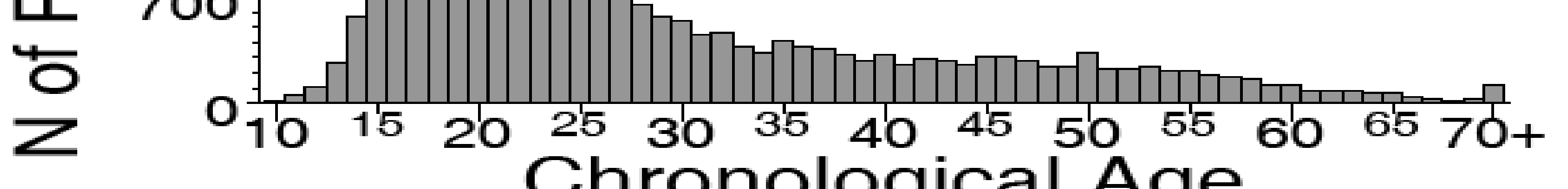

Subjective Age

Randomly-ordered items assessing subjective age

(response scale: $1-115$ years):

Felt age:

"How old do you feel?"

- Desired age:

"If you could choose, what age would you be?"

\section{Results}

In separate regressions with chronological age chronological age. For each 10-year increase in respondents' chronological age, felt age was only $6.4 \pm .03$ years older $\left(\mathrm{R}^{2}=.45\right)$ and desired age only 5.2 .03 years older $\left(\mathrm{R}^{2}=.52\right)$. On average, those under 25 felt older and desired to be even older than reality, while adults (aged 30+) felt younger and desired to be even younger.

Adolescents and young adults (10-25) felt older han their chronological age $(M=1.3$ years

older), with 34\% feeling older and $22 \%$ feeling Jounger than actuality. In contrast, adults aged 65-89 felt an average of 15.2 years younger, and most $(85 \%)$ felt younger than their chronological age, just $3 \%$ felt older. Alhough previous (Rubin \& Berntsen, 2006) emphasized the diferences between reporting same or

列 (13\% reported feeling their current age.
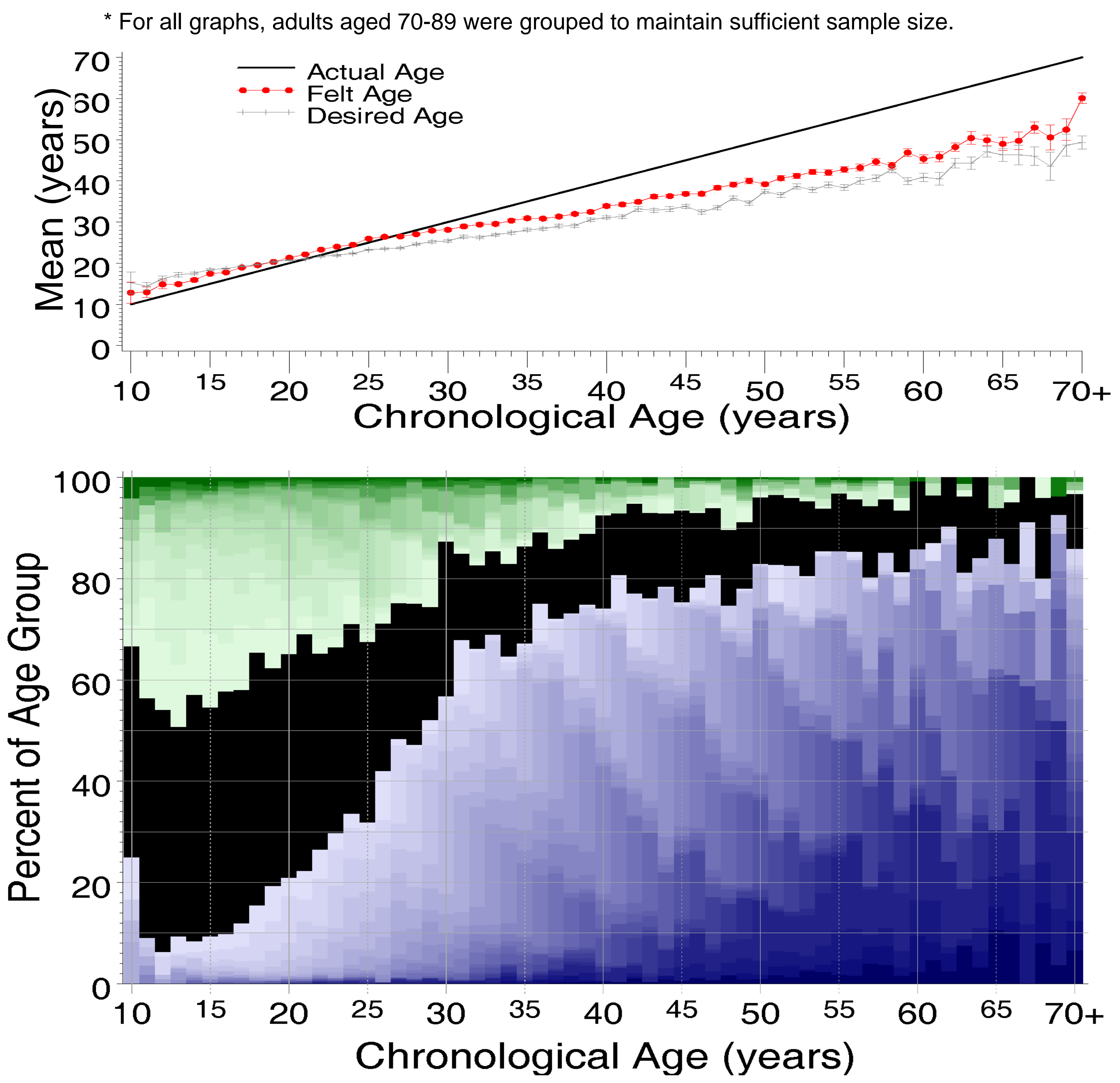

Same Age:

Chronological Age (years)

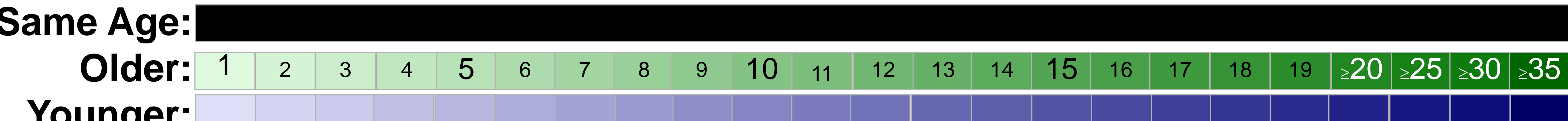

Younger:

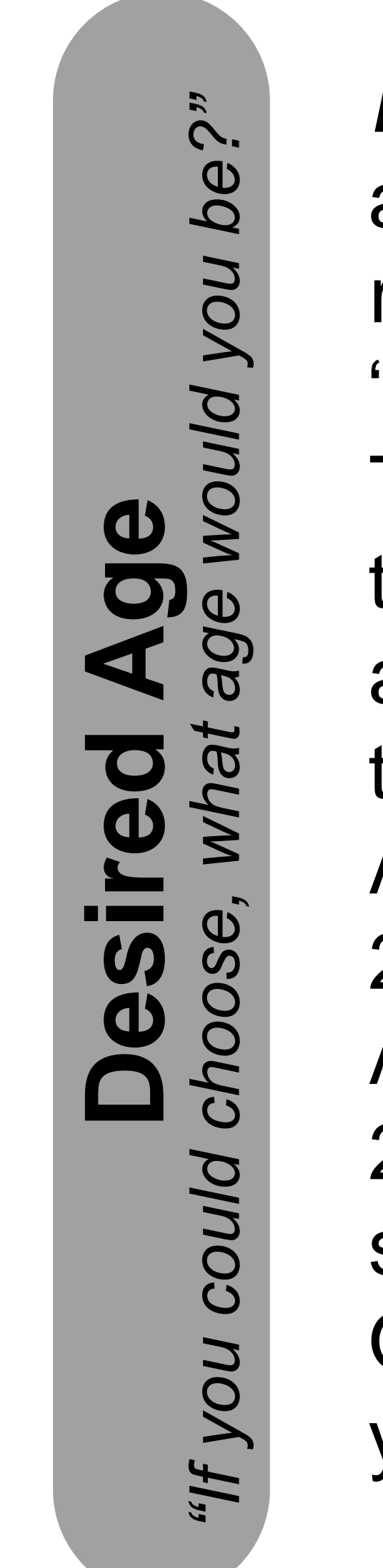

Desired age grew older along with chronological ge, but the discrepancy increased among older espondents. Respondents also tended to choose "landmark" desired ages (e.g., 21, 40,45). "Ideal age" for people can be conceived of as the at which people choose their current age 作 And A3\% of any other age desired their current age. Adolescents (ages 10-17, $M_{\text {ditt }}=2.8$, modal age = 1) and young adults (ages 18-25) desired to be lightly older $\left(M_{\text {diff }}=0.34\right.$ years, modal age $\left.=21\right)$ ged 65-89) desired

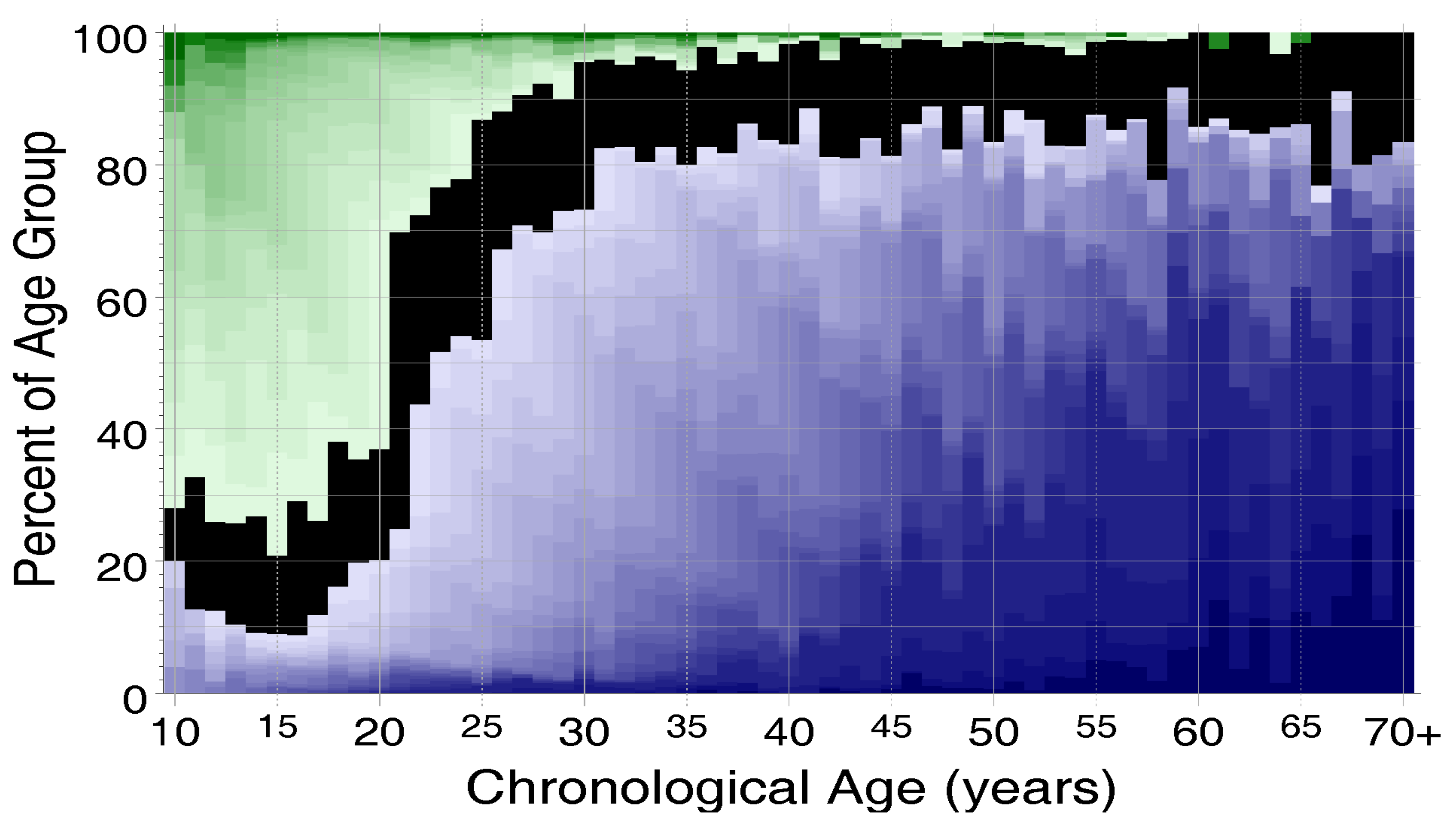

Results: con't.

Previous evidence for gender differences in subjective age has been mixed (Montepare \& Lachman, 1989; Rubin \& Berntsen, 2006). Women reported desired ages that were cher to their chronological age than men $(2.26 \pm .20$ years closer), and men's tendency to report a more youthful desired age increased with age (by $1.1 \pm .07$ years for every (a) ave. These results suggest hat he common praclice of gender differences and other patterns of change. Although the relationship between subjective age identity A che the cumulative barplots (Figures 2 \& 3) display how the

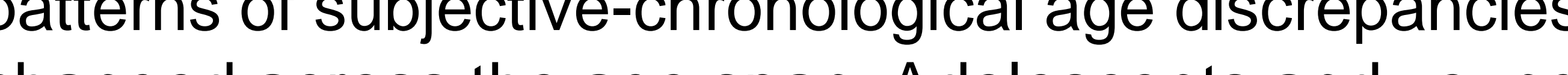
alts predoss the age span. Adolescents and young came are or a bit older, while middle and desired to be the

\section{Conclusions}

With each passing Earth year, felt and desired age do grow older, it just takes longer for the year to go by. Past age 25 ro so, subjeclve aging appears to occur on Mars, whe 25 sense, our minds age more slowly than our bodies do.

The results for desired age suggest that the turning point when chronological age begins to outpace subjective age is age 21. At least in American society, this age symbolizes sug access to the rights and privileges of adullhood, this suggests despite the heterogeneity in the timing of adult independes (Anett 2000), specific normative milestones may play an important role in subjective age identity.

At the same time, the linear increase in felt and desired ages suggests that mental experience of time - at least in terms of our personal experience - runs at a slower pace



